\title{
Oscillator clustering in a resource distribution chain
}

\author{
Postnov, D.; Sosnovtseva, Olga; Mosekilde, Erik
}

Published in:

Chaos

Link to article, DOI:

$10.1063 / 1.1852151$

Publication date:

2005

Document Version

Publisher's PDF, also known as Version of record

Link back to DTU Orbit

Citation (APA):

Postnov, D., Sosnovtseva, O., \& Mosekilde, E. (2005). Oscillator clustering in a resource distribution chain. Chaos, 15(1), 013704. https://doi.org/10.1063/1.1852151

\section{General rights}

Copyright and moral rights for the publications made accessible in the public portal are retained by the authors and/or other copyright owners and it is a condition of accessing publications that users recognise and abide by the legal requirements associated with these rights.

- Users may download and print one copy of any publication from the public portal for the purpose of private study or research.

- You may not further distribute the material or use it for any profit-making activity or commercial gain

- You may freely distribute the URL identifying the publication in the public portal

If you believe that this document breaches copyright please contact us providing details, and we will remove access to the work immediately and investigate your claim. 


\title{
Oscillator clustering in a resource distribution chain
}

\author{
Dmitry E. Postnov \\ Physics Department, Saratov State University, Astrakhanskaya Str. 83, Saratov, 410026, Russia \\ Olga V. Sosnovtseva ${ }^{a)}$ and Erik Mosekilde \\ Department of Physics, The Technical University of Denmark, 2800 Kgs. Lyngby, Denmark
}

(Received 4 August 2004; accepted 7 December 2004; published online 25 February 2005)

\begin{abstract}
The paper investigates the special clustering phenomena that one can observe in systems of nonlinear oscillators that are coupled via a shared flow of primary resources (or a common power supply). This type of coupling, which appears to be quite frequent in nature, implies that one can no longer separate the inherent dynamics of the individual oscillator from the properties of the coupling network. Illustrated by examples from microbiological population dynamics, renal physiology, and electronic oscillator theory, we show how competition for primary resources in a resource distribution chain leads to a number of new generic phenomena, including partial synchronization, sliding of the synchronization region with the resource supply, and coupling-induced inhomogeneity. @ 2005 American Institute of Physics. [DOI: 10.1063/1.1852151]
\end{abstract}

Synchronization of interacting oscillators is of interest in many areas of science and technology. The purpose of this paper is to investigate the special type of synchronization phenomena that arise in systems of coupled selfsustained oscillators where the interaction takes place via a competition for primary resources. This implies that one can no longer separate the characteristic modes of the individual oscillators from the properties of the coupling network. Our investigation is motivated by an attempt to understand how the distribution of blood in the renal arteriolar network interacts with the pressure and flow regulation of the individual nephron. However, to illustrate the generic aspects of the problem we also discuss examples from electronics and microbiological population dynamics. We show how sharing of resources can lead to coupling-induced inhomogeneity among the oscillators, and how this coupling structure tends to produce anti-phase synchronization.

\section{INTRODUCTION}

The classical synchronization paradigm ${ }^{1,2}$ considers the interaction of two or more oscillators, each with their own sources of energy, and with the coupling being responsible for the frequency entrainment and mutual amplitude adjustments. Well-studied coupling structures include local coupling, ${ }^{3}$ where oscillators in a lattice (or some other spatial arrangement) interact with their nearest neighbors, and global coupling, ${ }^{4}$ in which each oscillator in an ensemble interacts with all other oscillators (or with a mean field produced by these oscillators). The first type of coupling may represent the interaction of heart muscle cells or pancreatic $\beta$-cells via gap junctions ${ }^{5}$ through which ions and small molecules can pass from one cell to its neighbors. This coupling typically produces waves or pulses that propagate across the interacting units. Global coupling may be realized for a system of

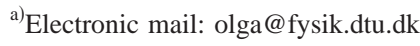

coupled electrochemical oscillators ${ }^{6}$ or for the metabolic oscillations in a suspension of yeast cells. ${ }^{7}$ Typical phenomena associated with this coupling structure are global synchronization, oscillator death through mutual suppression, and various forms of clustering in which the ensemble splits into subgroups of synchronized oscillators, but such that each subgroup maintains its own dynamics. More recently, the study of so-called small-world networks has attracted considerable interest. ${ }^{8,9}$ Here, the interaction among the oscillators combine a local coupling with a few (more or less random) long-range connections.

For all off the above coupling structures, the mathematical description assumes that the nonlinear properties of the individual functional unit (i.e., its natural frequency, its resistance to external perturbations and, if accounted for, its inherent source of noise) are governed by the unit's own parameters while the interaction is specified through a separate set of parameters that characterize the coupling structure and interaction strengths. Hence, one can distinguish the natural modes of the individual oscillators from the properties of the coupling network.

However, there is a variety of problems in physics, chemistry, biology, and engineering that cannot be considered within this paradigm, namely such problems where the coupling between the oscillators takes place via the distribution of the energy (or resources) that allows the individual oscillator to maintain its dynamics.

Typical examples, that we shall consider in the present paper include:

(i) Cascaded electronic oscillator circuits where the oscillators are coupled via a common power supply;

(ii) microbiological population dynamic systems where pools of interacting species couple via the flow of primary resources; and

(iii) renal flow and pressure regulation where nephrons in a vascular tree share a common blood supply. 
Our aim is not to discuss each of these problems in detail but to point to a number of generic phenomena associated with the particular coupling structure and to illustrate the spectrum of different areas where coupling via the distribution of primary resources takes place.

The system of cascaded electronic oscillators is of interest both because the governing equations are wellestablished and because detailed experimental verification of the simulation results are easy to obtain.

Microbiological population dynamics play an important role in many biotechnological industries. The homogeneous, well-controlled bacterial cultures used in modern cheese production, for instance, are often quite sensitive to virus attack, and considerable effort is devoted to the search for more resistant cultures. ${ }^{11}$ Based on original investigations by Levin et al. ${ }^{10}$ Baier et al. ${ }^{12}$ formulated a multispecies model of interacting bacteria-virus populations and studied the development of a chaotic hierarchy as the number of bacterial variants increased. A resource distribution chain with several cascaded pools was first considered by Postnov et al. ${ }^{13}$ They showed how the forcing of one habitant by the variation in substrate concentration in the overflow from the upstream habitant can produce increasingly complicated dynamics and how regions of phase synchronization could arise along the chain. In the present paper, these results will be placed in a more general perspective.

Nephrons are the functional units of the kidney in which urine is formed through a complicated balance of filtration, secretion and (active and passive) reabsorption processes. To protect its function against changes in the arterial blood pressure, the individual nephron disposes of a feedback mechanism (the tubuloglomerular feedback TGF mechanism) that produces a contraction of the afferent arteriole and, hence, a reduction of the incoming blood flow in response to elevated $\mathrm{NaCl}$ concentrations in the fluid leaving the nephron. The TGF regulation can generate self-sustained oscillations in the proximal intratubular pressure with a typical period of $30-40 \mathrm{~s} .{ }^{14}$ While for normal rats the oscillations have the appearance of a limit cycle, highly irregular oscillations are observed for spontaneously hypertensive rats. The tubulorglomerular feedback mechanism may be considered as reinforcement of the normal myogenic mechanism by which the smooth muscle cells in the arteriolar wall respond to changes in the transmural pressure. ${ }^{15}$

Nephrons are arranged in a tree structure with the afferent arteriole branching off from a common interlobular artery. This structure allows a change in the blood flow to one nephron to influence the blood flow to all the other nephrons in the tree. Interaction between adjacent nephrons can occur due to vascularly propagated coupling mediated by electrochemical signals and muscular contractions that travel along the arteriolar wall ${ }^{16}$ and hermodynamic coupling by which an increased flow resistance in the afferent arteriole leading to one nephron forces a higher blood flow to the neighboring nephrons. Only a few studies of multinephron systems appear to be available. ${ }^{17}$ We present a dynamical approach to the description of a vascular-nephron tree.

A typical feature of the considered examples is that the energy (or primary resources) available to the individual sub-
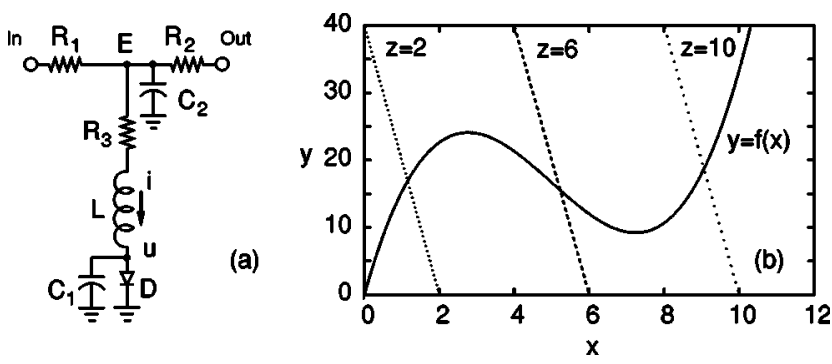

FIG. 1. (a) Circuit diagram and (b) the null-clines for the 2D limit case $R_{1,2}=0$. In the dimensionless model equations (1), $x$ represents the voltage $u$ across the tunnel diode $D, y$ is the current $i$ in the inductor $L$, and $z$ is the voltage $E$ across the capacitor $C_{2}$.

unit (and, hence, its mode of behavior) depends on the energy consumed by all the other oscillators in the system, both with respect to its average value and with respect to its temporal variation (amplitudes and phases). If the system has a cascaded structure, the energy supply tends to decrease along the chain. Hence, the functional units will operate in different regimes and, even if their parameters are identical, their amplitudes and frequencies may differ. Moreover, only a certain group of oscillators within a chain may experience the proper conditions to oscillate and/or synchronize, and this group may shift up and down along the chain depending on the total energy supply. The considered aspects of the problem are different from known examples of synchronized cluster formation in a chain of Rössler systems with gradually varying parameters. ${ }^{18}$

\section{FORMATION OF SPATIAL OSCILLATORY PATTERNS IN RESOURCE-DEPENDENT SYSTEM}

\section{A. Model}

To investigate the typical behavior patterns in a resource consumption chain we need a simple model that mimics the main properties of such a system. Let us consider the electronic circuit with a tunnel diode shown in Fig. 1. Here, the incoming $I_{\text {in }}$ and outgoing $I_{\text {out }}$ power supply currents are explicitly taken into account. The voltage $E$ plays the role of energy source for the oscillator system containing the elements $R_{3}, L, C_{1}$, and $D$. Self-sustained oscillations are maintained due to the $N$-shaped characteristics (negative differential resistance) of the diode $D$. The oscillation period is determined by the capacitance $C_{1}$ and the inductance $L$. Capacitor $C_{2}$ is introduced to account for possible accumulation of energy by an individual oscillator while the resistor $R_{1}$ is responsible for the finite replenishment rate and for losses because of transmission.

Using Kirchhoff's law for the circuit in Fig. 1(a) and introducing the new time variable $\tau=t / C_{1}$ we can write down the governing equations in dimensionless form

$$
\dot{x}=y-f(x),
$$

$\varepsilon \dot{y}=z-y R-x$, 


$$
\gamma \dot{z}=-y+\frac{1}{r}\left(e_{\text {in }}-2 z+e_{\text {out }}\right) .
$$

Here, $\varepsilon=L / C_{1}$ and $\gamma=C_{2} / C_{1}$. $e_{\text {in }}$ and $e_{\text {out }}$ are potentials in the "in" and "out" points, respectively. Parameters $r$ and $R$ are dimensionless representations of the resistors $R_{1}=R_{2}$ and $R_{3}$. Variables $x, y$, and $z$ correspond to the voltage $u$ over the diode, the current $i$ through the inductor $L$, and the voltage $E$ on the capacitor $C_{2}$, respectively. We assume that $C_{2} \ll C_{1}$ so that $z$ quickly follows variations of $x$ and $y$. In the following calculations $\varepsilon=0.1$ and $\gamma=0.01 . f(x)$ is assumed to be of cubic shape with the nonlinearity chosen in the form:

$$
f(x)=1 / 3+20 x-5 x^{2}+x^{3} / 3
$$

In the limit $r \rightarrow 0$, one can get $z=\left(e_{\text {in }}+e_{\text {out }}\right) / 2$. Hence, we obtain a 2D oscillator which is similar to the FitzHughNagumo model ${ }^{19}$ with $z$ as a control parameter. Figure 1(b) illustrates the location of the nullclines in the system. Note that the $x$-nullcline coincides with the $f(x)$ function. It is clearly seen that for small and large values of $z$, intersection of the nullclines occurs outside of the interval with negative slope of $f(x)$, i.e., the equilibrium point is stable. For intermediate values of $z$, there is a couple of Hopf bifurcation points where a stable limit cycle is born, and extinguished.

In order to build a one-dimensional array of such functional units we take

$$
e_{\text {in }}=z_{j-1}, \quad e_{\text {out }}=z_{j+1}, \quad j=1 \ldots N,
$$

where $j$ represents the number of the oscillator, and $N$ is the total number of units. $z_{0}$ is constant bias voltage, hereafter denoted as $Z_{0}$. Free end of the chain is modeled by $z_{N+1}$ $=z_{N}$.

\section{B. Clustering}

Organized in a chain, the units (1) become globally coupled via variation of the $z_{j}$ variables. There is a gradual decrease of the mean value of $z_{j}$ along the chain because of the voltage drop across each coupling resistor $r$. Note that the current along the chain splits into two currents at each unit. Thus it decreases along the chain, and the drop of $z_{j}$ from unit to unit becomes smaller and smaller.

In the phase space of the whole system, the variation of the mean value of $z_{j}$ affects the stability of the global equilibrium state that can be defined from

$$
\dot{x}_{j}=0, \quad \dot{y}_{j}=0, \quad \dot{z}_{j}=0, \quad j=1 \ldots N .
$$

The transition from damped behavior to self-sustained oscillations for a particular unit in the chain takes place through an Andronov-Hopf bifurcation in the $\left(x_{i}, y_{i}\right)$ phase sub-space. In Fig. 2 the real part for each pair of complexconjugated eigenvalues is plotted against $Z_{0}$ for a chain of 10 units (1). The third eigenvalue for each subsystem is not shown in the figure since it is strongly negative in the whole range of control parameters. With increasing $Z_{0}$ from 4.0 , the eigenvalues are seen one by one to cross the imaginary axis and attain positive real values and as $Z_{0}$ increases further, again to become negative. According to the number of eigenvalues with positive real part, the dimension of the unstable

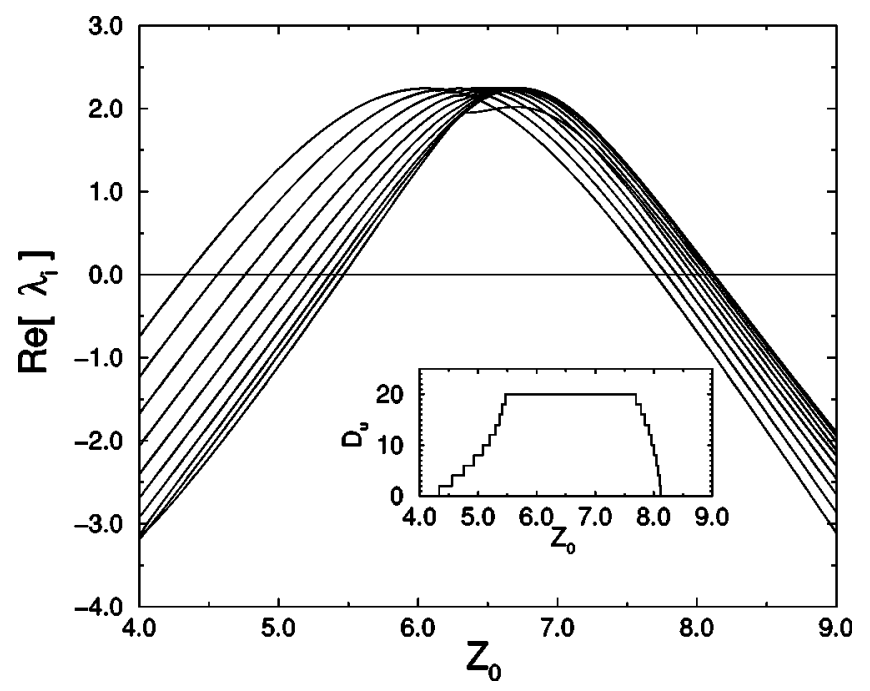

FIG. 2. The real part of the equilibrium state eigenvalues $\lambda_{i}$ exceeds zero in a certain range of $Z_{0}$ (each curve represents one pair of complex-conjugated eigenvalues). The resulting dimension of the unstable manifold $D_{u}$ vs $Z_{0}$ is shown in the insert.

manifold $D_{u}$ of the equilibrium point first raises and then decreases with increasing $Z_{0}$ (insert in Fig. 2).

From a physical point of view, these results imply the possibility of $D_{u} / 2$-mode self-sustained dynamics in the whole system. In spite of the fact that we can not formally assign a given pair of eigenvalues to a specific unit of the chain, it is clear that the first pair of eigenvalues crossing zero should be related to the first unit in a chain that receives the necessary energy input from $Z_{0}$. Similarly, the subsequent crossings of zero by different pairs of eigenvalues represent the subsequent transitions of oscillatory units from the damped state to self-sustained dynamics, or vice versa. This is the basis for the formation of a group of units along the chain, that we call an oscillatory cluster.

Let us now consider what happens in a longer chain of 50 units in terms of amplitudes of oscillations. In Fig. 3, the

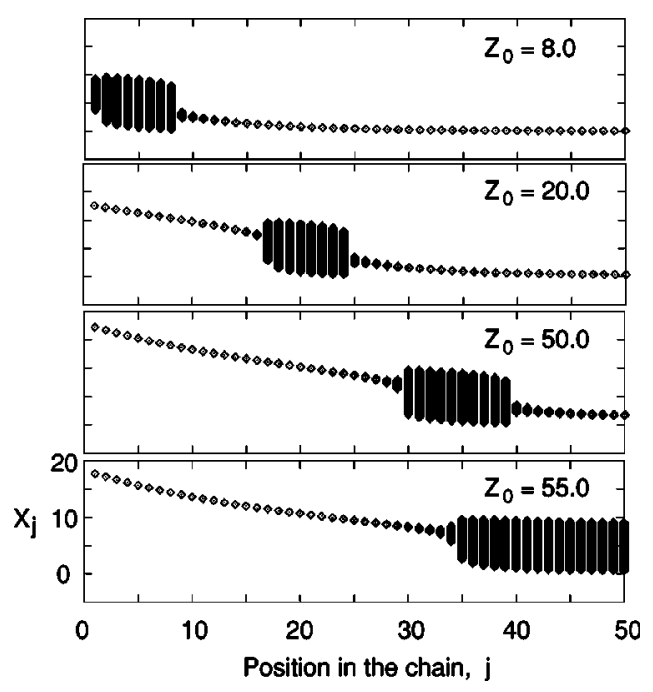

FIG. 3. With increasing $Z_{0}$, the oscillatory cluster changes its position along the chain and varies slightly in size. Parameters are fixed at $r=0.001, R$ $=0.05, \varepsilon=0.1$, and $\gamma=0.001$. 


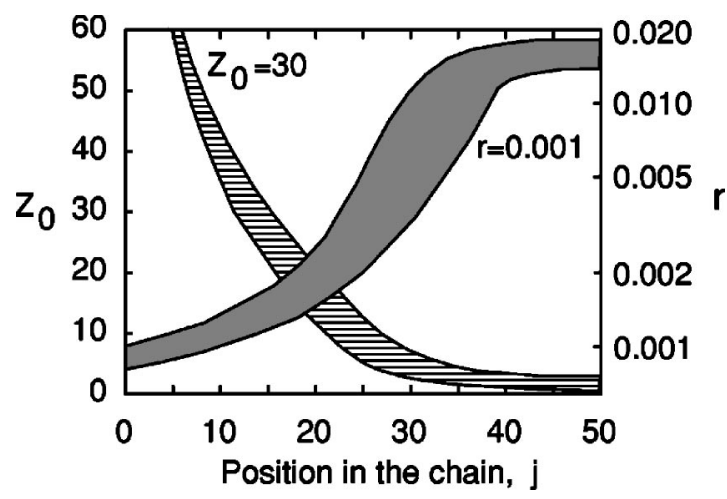

FIG. 4. The position and size of the oscillatory cluster are given by the gray region for different $Z_{0}$ values (at $r=0.001$ ), and by the hatched region for different $r$ values (at $Z_{0}=30$ ). Other parameters are fixed at $R=0.05, \varepsilon$ $=0.1$, and $\gamma=0.001$.

$x_{j}$ variable of each unit is plotted against its position in the chain for different voltages $Z_{0}$ of the power supply. For relatively small voltages $\left(Z_{0}=8.0\right)$, the first 8 units display oscillations of considerable amplitude. For the next units, the mean value of $z_{j}$ is not sufficient to support self-sustained dynamics.

For a larger value of the voltage $\left(Z_{0}=20.0\right)$, inspection of the figure shows that the first 16 units no longer display significant temporal variations of $x_{j}$ because the high mean value of $z_{j}$ places the individual unit in a damped state according to Fig. 1(b). The next 8 units demonstrate selfsustained dynamics while the rest are in a damped state. With increasing $Z_{0}\left(Z_{0}=50\right)$, the oscillating group shifts towards the end of chain and grows in size. Thus, we observe an oscillatory cluster shifting upstream or downstream the chain with variation of the energy source parameter $Z_{0}$. When approaching the low-voltage end of the chain, the cluster becomes fairly large before it completely disappears at $Z_{0}$ $\approx 59$.

While $Z_{0}$ defines the maximal level of $z_{j}$ for units in the chain, the parameter $r$ affects the voltage drop from unit to unit. Hence, $r$ can also influence the position and size of the oscillatory cluster. Figure 4 reveals the relation between the variations of $Z_{0}$ and $r$. In both cases, the size of the cluster remains relatively stable until the end of the chain is reached. Here the cluster becomes significantly longer before it disappears. This can partly be explained by the decreasing voltage drop per oscillator along the chain. When the cluster is located in the middle of a chain, the tailing oscillators consume electric power even though they are in a damped state. This produces an additional voltage drop and reduces the cluster size. Closer to the end of chain, the cluster has less "silent" energy consumption. The voltage drop from one unit to the next decreases, and the cluster length grows.

\section{Intra-cluster synchronization}

With the emergence of a cluster of oscillatory, identical units, one might expect to observe synchronized behavior for the globally coupled identical oscillators. However, inspection of the cluster reveals a completely different situation.
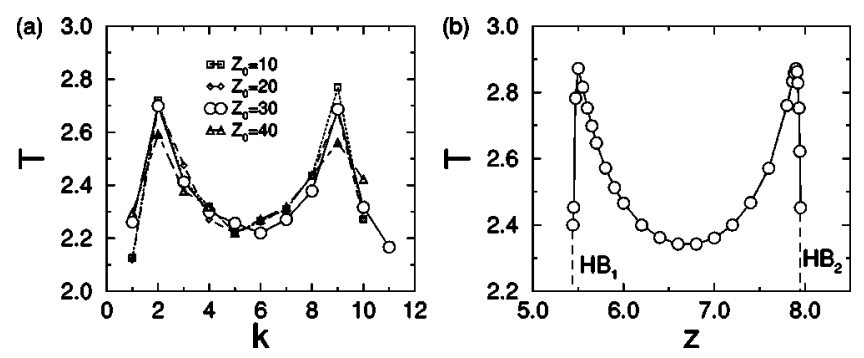

FIG. 5. (a): The distribution of oscillation periods $T$ inside the cluster is preserved, while the whole cluster moves with the variation of $Z_{0} . k$ represents the relative position within the cluster. At $r=0.001, R=0.05, \varepsilon=0.1$, and $\gamma=0.001$. (b) $T$ vs $z$ dependence for the individual oscillatory unit reveals the origin of intra-cluster period distribution.

Figure 5(a) shows how the mean period of oscillations is distributed along the cluster for different values of the voltage $Z_{0}$. In this figure $k=1$ is assigned to the first oscillator with self-sustained dynamics in the chain. Thus, different positions along the chain correspond to different curves in Fig. 5(a). This allows us to compare the spatio-temporal structure of clusters for different values of the control parameter. It is remarkable that the period distribution maintains its form. For all the values of $Z_{0}$ shown there are two oscillators with longer periods close to the beginning and the end of the cluster, and there is a clear minimum of the oscillation period near the center of the cluster. Both the first and the last oscillators in the cluster also display relatively low oscillation periods.

To explain the particular shape of the intra-cluster period distribution, we calculate how the period depends on the $z$ value for an individual system (1) in the limit $r \rightarrow 0$. The results are given in Fig. 5(b). The period distribution along the cluster clearly follows the behavior of an individual unit with decreasing $z$. The observed structure is the result of the drop in $z_{j}$ from unit to unit, combined with the variation of $f(x)$ in the region of differential negative resistance.

Hence, in spite of the presence of a coupling between all cluster units, synchronization is not observed due to a coupling-induced frequency mismatch between the oscillators. For small values of $r$ the frequency mismatch vanishes together with the drop of $z_{j}$ from one oscillator to the next. However, the coupling vanishes too. The larger $r$ is, the stronger the coupling will be, but at the same time the frequency mismatch becomes more pronounced between two neighboring units. Together this results in an asynchronous intra-cluster behavior in the considered parameter range.

As observed in Fig. 5(b), the maximal possible period drop between two units is not greater than $\Delta T \approx 0.53$ corresponding to the difference between the top and bottom points of the curve. Thus, the coupling-induced mismatch is limited to about $20 \%$, while the coupling strength can be increased by an appropriate choice of control parameters. Let us select $R=0.01$ and $r=0.02$. This provides a strong drop of $z_{j}$, and the oscillatory cluster consists mostly of just two units. The cluster position and operating regimes are schematically given in Fig. 6 together with representative phase plots. The synchronized pairs of oscillator units are given in gray, while 


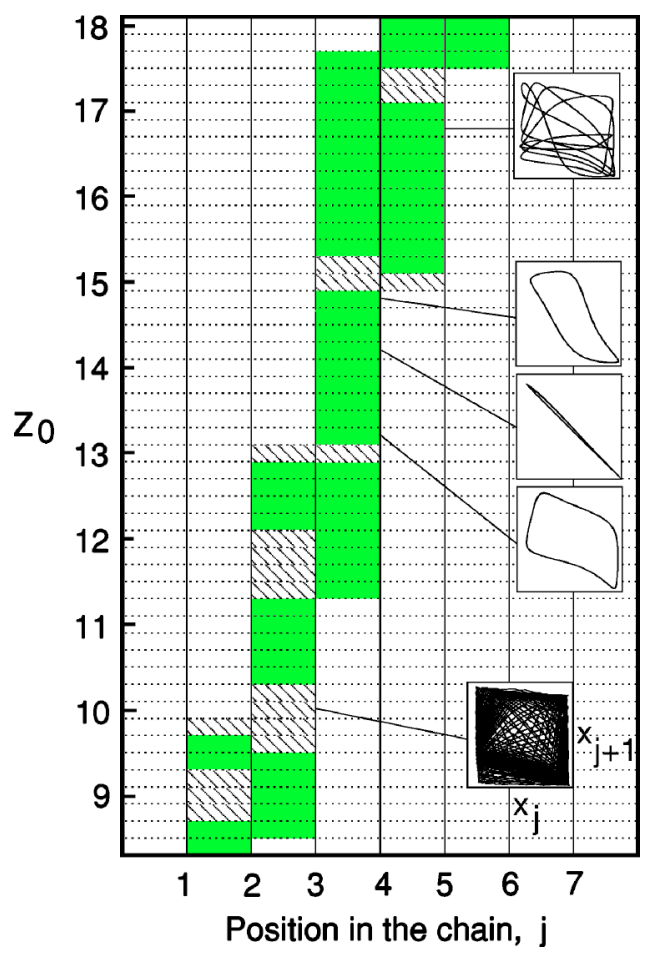

FIG. 6. Small-sized clusters exhibit different synchronous or asynchronous patterns with varying $Z_{0}$ for strong interaction along the chain $(r=0.02, R$ $=0.01, \varepsilon=0.1$, and $\gamma=0.001)$. Gray areas denote synchronized behavior, and hatched areas denote asynchronous dynamics.

asynchronous behavior is denoted by hatched regions. For higher values of $Z_{0}$ the oscillatory cluster moves out of the chain to the right.

For some intervals of $Z_{0}$ (e.g., $Z_{0}$ $\in[10.4,11.2],[12.2,12.8])$, oscillations in clusters of 2 or 3 units are synchronized with a phase lag between two neighboring units as shown in the inserts. Note, that the phase lag in the two-unit cluster increases with $Z_{0}$, and the cluster passes through the anti-phase state somewhere in the middle of the $Z_{0}$ interval for synchronous behavior. For the two-unit cluster there is a clear explanation: When only two units in the chain display self-sustained dynamics, the influence of other units can be considered as a shift of control parameters. Hence, we have a system of two oscillators coupled in a competitive way that typically leads to anti-phase synchronization. Together with the coupling induced frequency mismatch this provides anti-phase rather than in-phase synchronization. At some value of $Z_{0}$ we find the most balanced regime (minimal frequency mismatch), and the synchronization regime becomes anti-phase. The insert for the $Z_{0}=16.8$ shows that there is also synchronization at various rational frequency ratios.

At some values of $Z_{0}$ the cluster changes its position. Such a translation is typically accompanied by an extension of the cluster to 3 units and a desynchronization between two or all three involved units (see the bottom insert in Fig. 6). When the first or last element of a cluster passes through the Hopf bifurcation point [Fig. 5(b)], the coupling-induced frequency mismatch can be strong enough to desynchronize the intra-cluster behavior. We can thus conclude that: (i) Although the chain units are originally identical, the resource drop along the spatial coordinate introduces variations of the operating point and, hence, a frequency mismatch between neighboring units;

(ii) in the case of weak coupling, the cluster elements are generally out of synchrony, and the period distribution along the cluster follows the curve of period vs energy supply for the individual oscillatory unit. The resulting intra-cluster period/frequency distribution preserves its structure as the cluster moves along the chain with variation of the bias voltage. Due to the competitive nature of the coupling, there is a tendency for anti-phase synchronization;

(iii) for strong enough coupling, intra-cluster synchronization is typical. However, shifts of the cluster position are accompanied by desynchronization of the cluster elements.

\section{BIOLOGICAL EXAMPLES}

\section{A. Microbiological oscillators}

To explore how cluster formation manifests itself in a very different system we consider a microbiological chain of interacting bacteria and viruses. Being coupled via the flow of nutrition, such a population model fits well with the ideas of spatial development and damping of self-sustained dynamics.

\section{Model}

Our analysis considers a one-dimensional array of population pools as originally introduced by Postnov et al. ${ }^{13}$ Each pool is the habitat for a three-variable predator-prey system, consisting of bacteria, infected bacteria, and viruses, represented in the equations by their concentrations $B_{i}, I_{i}$, and $P_{i}$, respectively ( $i$ denotes the pool number). Nutrition balance of inflow, outflow, and consumption provides a fourth equation for the substrate concentration $S_{i}$. Altogether this leads to the following set of coupled differential equations

$$
\begin{aligned}
& \frac{d B_{i}}{d t}=\frac{\nu B_{i} S_{i}}{S_{i}+K}-\rho B_{i}-\alpha \omega P_{i} B_{i}, \\
& \frac{d I_{i}}{d t}=\alpha \omega B_{i} P_{i}-\rho I_{i}-I_{i} / \tau, \\
& \frac{d P_{i}}{d t}=\phi-P_{i} \rho-\alpha B_{i} P_{i}-\alpha I_{i} P_{i}+\beta I_{i} / \tau, \\
& \frac{d S_{i}}{d t}=\rho\left(S_{i-1}+\sigma_{i}\right)-\rho S_{i}-\frac{\gamma \nu B_{i} S_{i}}{S_{i}+K},
\end{aligned}
$$

where the term $\nu B_{i} S_{i} /\left(S_{i}+K\right)$ in the first and fourth equations describes standard Monod kinetics for bacterial growth. The Michaelis-Menten constant $K$ represents the concentration of nutrients at which the growth rate is reduced to half its maximal value, and each cell division is assumed to be associated with a resource consumption $\gamma$. For all variables, negative 
(a)
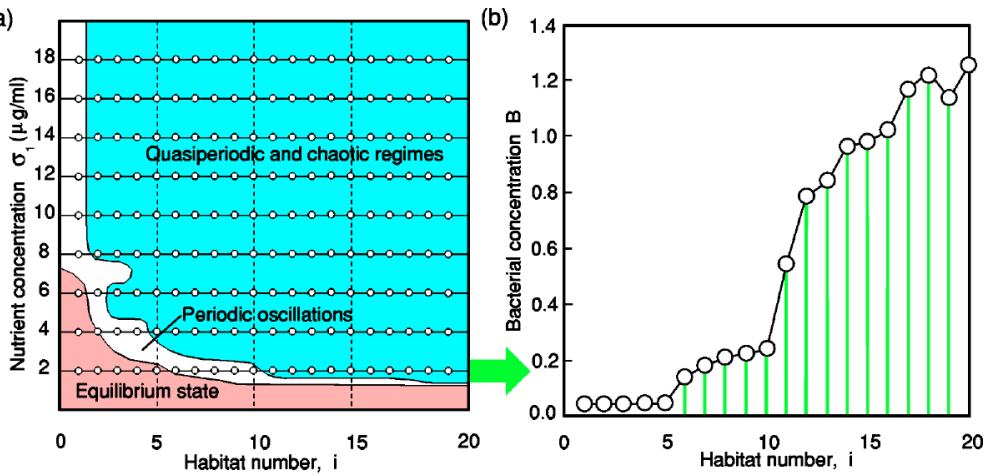

(c)

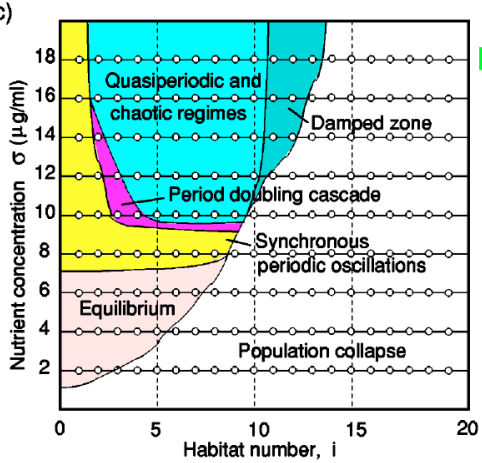

(d)

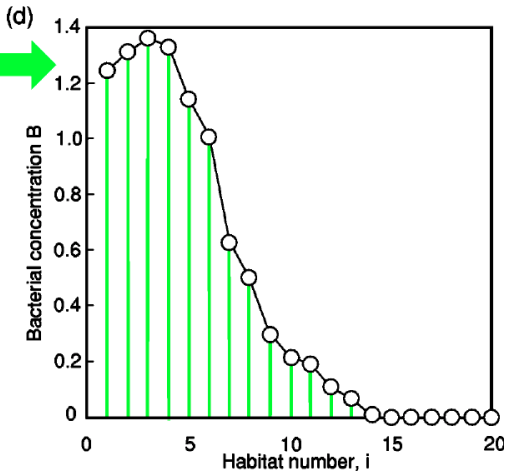

FIG. 7. Overview of the behavior of a chain of 20 population pools: Diagram of dynamical regimes (left panels) and variance of bacterial concentrations for selected $\sigma$ values (right panels). $i$ is the pool number along the chain and $\sigma_{i}$ the concentration of the local nutrient supply. (a) and (b) lateral nutrition: $\sigma$ is assumed to be the same for all pools; (c) and (d) upstream nutrition: $\sigma_{1}>0, \sigma_{i}=0, i=2,3,4, \ldots 20$. terms proportional to $\rho$ in the governing equations reflect the washing out from the habitat. According to our assumptions, however, only nutrients will be transmitted to the next pool. Infection of bacteria by viruses is described by the term $\alpha \omega B_{i} P_{i}$ in (3) and (4). Here, $\alpha$ is the kinetic rate constant, and $\omega$ is the probability that a virus particle successfully infects a cell, once it has affixed to its surface. The $I_{i} / \tau$ term in (4) and (5) describe a lytic response to the virus attack where, after a latent period $\tau$ of the order of $30 \mathrm{~min}$, the infected cell bursts and releases an average of $\beta$ new viruses. Note, the term $-\alpha I_{i} P_{i}$ in (5) represents unsuccessful virus attacks on already infected cells. Coupling between the pools takes place only through the flow of nutrients with a total incoming rate of $\rho\left(S_{i-1}+\sigma_{i}\right)$, an outflow of $-\rho S_{i}$, and a consumption in the habitat of $\gamma \nu B_{i} S_{i} /\left(S_{i}+K\right)$. For the first habitat, $S_{i-1} \equiv S_{0}$ is assumed to be zero. $\sigma_{i}$ represents a possible lateral nutrition source for the $i$ th habitat.

The parameter values that we have applied in the present analysis correspond to the values used in our previous studies: $^{12} \nu=0.024 \mathrm{~min}^{-1}, K=10 \mu \mathrm{g} / \mathrm{ml}, \tau=30 \mathrm{~min}, \omega=0.8$, $\gamma=0.01 \mathrm{ng}, \beta=100$. These values are also in general agreement with the experimental values obtained by Levin et al. ${ }^{10}$ for particular strains of bacteria and viruses. The concentrations $B_{i}, I_{i}$, and $P_{i}$ will be specified in units of $10^{6} / \mathrm{ml}$. Hence, we have used a value of $\alpha=10^{-3} \mathrm{ml} / \mathrm{min}$ (as compared with the value $\alpha=10^{-9} \mathrm{ml} / \mathrm{min}$ applied by Baier et al. ${ }^{12}$ ).

Like many other ecological models, our system involves positive feedback mechanisms related to the replication of bacteria and viruses. There are nonlinear constraints associated both with the bacterial growth rate and with the infection rate, and there is a delay associated with replication of the viruses. The rate of dilution is a main determinant of dissipation in the system. In the absence of viruses, the single pool model displays an equilibrium point

$$
B_{0}=\frac{1}{\gamma}\left(\sigma-\frac{\rho K}{\nu-\rho}\right), \quad S_{0}=\frac{\rho K}{\nu-\rho},
$$

in which the rate of bacterial growth balances the wash out. For dilution rates $\rho>\rho_{c}=\sigma \nu /(K+\sigma)$, only the trivial equilibrium point $B_{1}=0, S_{1}=\sigma$ exists.

As $\rho$ is reduced below $\rho_{c}$, the equilibrium population of bacteria starts to increase. At the beginning, the cell concentration is still too small for an effective replication of viruses to take place, and the virus population remains nearly negligible. As the dilution rate continues to decrease, however, the virus population grows significantly. The model then undergoes a Hopf bifurcation, and the system starts to perform self-sustained oscillations.

\section{Spatial dynamics}

Depending on the population sizes attained in the upstream habitats, different degrees of depletion of the nutrient concentration will occur, and as the surplus resources continue to flow into the next habitat, this population pool will be modulated by a temporal nutrient supply that depends on the type of dynamics realized in the former pool.

Along the chain there will be a net consumption of resources. However, different choices of the surplus nutrient supply $\sigma_{i}$ along the chain allow us to simulate different patterns of growth dynamics. Below we consider two important cases: Lateral nutrition [Figs. 7(a) and 7(b)] and afferent nutrition [Figs. $7(\mathrm{c})$ and $7(\mathrm{~d})]$. The dilution rate is assumed to be $\rho=0.003 / \mathrm{min}$, and the nutrient concentration is specified in $\mu \mathrm{g} / \mathrm{ml}$. 
In the first case, the choice of equal values of $\sigma_{i}=\sigma, i$ $=1,2,3 \ldots$ provides a separate influx of resources to each habitat. For low values of $\sigma$, the system attains a stable equilibrium state that extends along the entire chain. As the nutrient supply is increased, starting in the downstream end of the chain, habitat after habitat begins to perform selfsustained oscillations. There is an interval where all habitats from a given number and up execute a synchronous periodic behavior. As $\sigma$ is further increased, the downstream habitats begin to show quasiperiodic, chaotic and higher order chaotic behaviors of increasing complexity, and only the intermediate or first pools perform simple periodic oscillations. For $\sigma>7 \mu \mathrm{g} / \mathrm{ml}$, only the motion of the first habitat remains periodic. Figure 7(b) shows the variation of the bacterial concentration $B$ along the chain for $\sigma=2.0 \mu \mathrm{g} / \mathrm{ml}$. It is clearly seen that the first 5 pools reach an equilibrium state at very low $B$ values. The bacterial populations survive here, but the concentrations are not high enough to generate selfsustained oscillations. Oscillatory behavior with increasing amplitude (gray lines indicate the variance of $B_{i}$ ) is observed starting from the 6th habitat. Pools from 6 to 10 form a cluster with synchronized periodic oscillations. With gradually increasing resources, oscillations become chaotic in the 11th habitat and maintain this dynamics till the last, 20th, habitat.

A second interesting case is afferent nutrition where there is a single source of nutrition to the first habitat [Figs. $7(\mathrm{c})$ and 7(d)]. Since each bacterial population consumes part of the incoming resources, the concentration decreases along the chain. Thus, for some habitat the available resources will not suffice to support self-sustained oscillations. However, the modulation of $S$ may still be propagated with the flow providing an oscillatory forcing for habitats in the rest chain. In this case, one can observe a limited region of selfsustained oscillations along the chain [Fig. 7(c)].

For low values of $\sigma_{1}$, a number of habitats at the beginning of a chain may be able to attain fairly high levels of bacterial concentration. However, these concentrations are not sufficient to produce self-sustained dynamics in interaction with the viruses. As more and more of the resources are consumed along the chain, the bacterial populations collapse $\left(B_{i} \approx 0\right)$. At $\sigma_{1}=7.024 \mu \mathrm{g} / \mathrm{ml}$ the first population reaches the point of Hopf bifurcation and self-sustained dynamics arises in the chain. Since the variation of the natural frequency is weak and the coupling strength (proportional to modulation depth of resources flow) is quite high, one observes synchronization in the group of habitats that display self-sustained dynamics.

Further increase of $\sigma_{1}$ to $10.0-12.0 \mu \mathrm{g} / \mathrm{ml}$ reveals a different pattern where only the first two habitats maintain the regime of synchronous regular oscillations, while perioddoubled regimes and the development of chaos can be observed further downstream.

Finally, for $\sigma_{1}>16.0 \mu \mathrm{g} / \mathrm{ml}$ only the first habitat shows regular oscillations (because this is the only possible oscillating regime for an individual system), while the subsequent are in a chaotic state. The case $\sigma_{1}=18.0 \mu \mathrm{g} / \mathrm{ml}$ is illustrated in Fig. 7(d). By virtue of the resource consumption along the chain, the self-sustained chaotic behavior dies out after the 10th habitat. However, the next three habitats display some intermediate dynamical patterns, representing neither the self-sustained regime nor the population collapse. We can describe these states in terms of chaotic forcing across the Hopf bifurcation point for the individual system. Thus, such habitats switch chaotically between a self-sustained regime when the nutrition amount temporarily is high enough, and a damped state. In Fig. 7(d) habitats 11, 12, and 13 display low, but finite amplitudes of variation in the bacterial concentrations. All downstream habitats are in the population collapse regime $\left(B_{i} \approx 0\right)$.

Let us summarize some of the main findings:

(ii) inside such clusters, subclusters with different behavior (steady state, regular synchronous oscillations, or chaotic behavior) can be detected. Note that chaotic regimes can also be synchronous or asynchronous as described by Postnov et al.; ${ }^{13}$

(iii) there is an intermediate operating regime arising via periodic or chaotic modulations of the individual system across a Hopf bifurcation point.

\section{B. Renal autoregulation}

While a chain of microbiological habitats provides a one-way coupling between the oscillators, the next example involve more complicated distribution network associated with an asymmetric but global coupling through the sharing of blood flow in a nephronic system.

The blood filtration in kidney is processed with a large number of sub-units (nephrons) connected to a complex branching structure of vessels called the preglomerular vascular tree with an inhomogeneous distribution of arteriolar lengths, nephron parameters, etc. ${ }^{20}$ Since the individual nephron is known to operate in a regime of self-sustained oscillations with the arterial pressure being a control parameter, the coupled nephrons can be considered in the framework a resource distribution system.

\section{Vascular-nephron model}

In order to examine the typical mechanisms associated with a structure of preglomerular vascular tree we consider a simplified vascular network that allows us to made conclusions about the main operating regimes and the transitions between these regimes with varying parameters.

Our model for the vascular-nephron tree consists of a set of afferent arterioles branching off from a single interlobular artery as shown schematically in Fig. 8. The vascularnephron tree structure is described in terms of the lengths of the arteriolar and arterial branches together with their hemodynamic resistances. It is assumed that the glomerulus of each nephron is connected to the corresponding branching point via an arteriole of length $L_{i}^{g}$, and of hemodynamic resistance $R_{i}^{g}, i=1 \ldots 12$. The arterial pressure $P_{a}$ to be used in the model of individual nephron now becomes the driving 

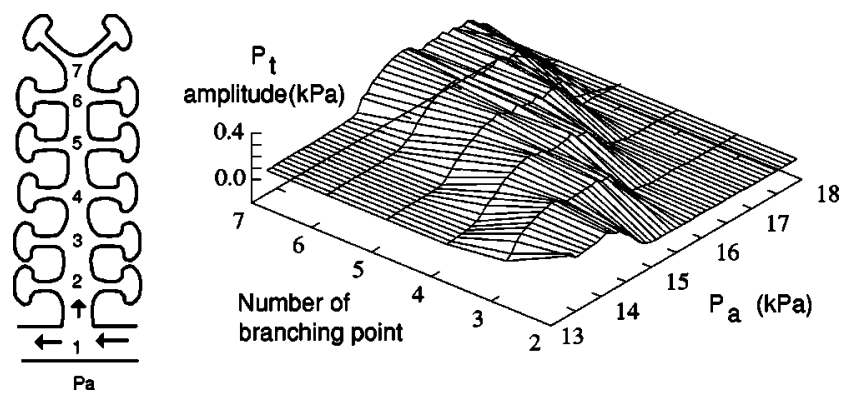

FIG. 8. Left: Sketch of vascular-nephron tree including the interlobular artery, the afferent arterioles and the glomeruli. Right: Oscillation amplitude as a function of arterial pressure and position of the branching point along the vascular tree.

blood pressure at the given branching point $P_{j}^{b}, j, k=2, \ldots 7$. Connection between the branching points is described in terms of branch lengths $L_{j k}^{b}$ and their hemodynamic resistances $R_{j k}^{b} j=1 \ldots 7$. The same approach is used to describe the connection of branching point 1 to the terminal points with the constant pressure values $P_{\text {in }}$ and $P_{\text {out }}$. This part of the vascular-nephron tree imitates the connection of the tree to higher-level arteries. To describe a drop of blood pressure along the vascular-nephron tree the transient time for blood pressure distribution over all branching points is assumed to be much smaller than the characteristic time scale for the TGF regulation in the nephrons. In other words, the elasticity parameter of the arteriolar walls is considered to be so high that we can neglect time delays in the pressure evolution. With this approach we can calculate the static pressure distribution for any state of connected nephrons using linear algebraic equations written for each branching point. An example of such an equation for the 6th branching point of the vascular-nephron tree (Fig. 8) reads:

$$
\frac{P_{5}^{b}-P_{6}^{b}}{R^{b}}-\frac{P_{6}^{b}-P_{7}^{b}}{R^{b}}+\frac{P_{3}^{g}-P_{6}^{b}}{R_{3}^{g}}+\frac{P_{4}^{g}-P_{6}^{b}}{R_{4}^{g}}=0 .
$$

Here, $P_{3}^{g}$ and $P_{4}^{g}$ represent the blood pressure in the glomerulus of 3th and 4th nephron. $P_{5}^{b}, P_{6}^{b}$, and $P_{7}^{b}$ represent the blood pressure in the 5,6 , and 7th branching point, respectively. $R^{b}$ denotes the hemodynamic resistance between 6th and 7th branching points. This resistance is assumed to be the same for all branches. $R_{3}^{g}$ and $R_{4}^{g}$ are the hemodynamic resistances to 3th and 4th nephron, respectively. Note that $R_{3}^{g}$ and $R_{4}^{g}$ are not constant because it includes the resistance of an active part of an afferent arteriole.

Equations of this type for the all branching points are obviously interdependent and, hence, produce a global hemodynamic coupling among nephrons in the vascular-nephron tree. The strength of this coupling is generally increasing with $R_{k j}^{b}$, but decreasing with increasing $R_{i}^{g}$.

Neighboring nephrons can also influence one another through a vascular propagated electrical (electrochemical) signal. To account for this mechanism, the total activation potential for $k^{\prime}$ th nephron in Eq. (12) is assumed to be the sum of contributions from all other nephrons in the tree. Moreover, the electrical activation potentials are assumed to propagate along the vascular wall with an exponential decay.
In this way, the vascular propagated interaction is delivered to each nephron as an additional part of its activation potential $\Psi$ :

$$
\Delta \Psi=\sum_{i=1, i \neq k}^{N} \Psi_{i} \exp \left(-\gamma\left(L_{j i}+L_{k}^{g}\right)\right),
$$

where $j$ is the number of the branching point to which the considered nephron with number $k$ is connected. The matrix $L_{j i}$ contains the lengths from a given branching point $j$ to all nephrons $i=1 \ldots N$, and $L_{k}^{g}$ is the length from the given nephron to the connected branching point.

Each individual nephron may be described by the following model: ${ }^{22,23}$

$$
\begin{aligned}
& \dot{P}_{t}=\frac{1}{C_{\mathrm{tub}}}\left\{F_{f}\left(P_{t}, P_{a}, r\right)-F_{\text {reab }}-F_{H}\right\}, \\
& \dot{r}=v_{r}, \\
& \dot{v}_{r}=\frac{1}{\omega}\left\{P_{\mathrm{av}}\left(P_{t}, P_{a}, r\right)-P_{\mathrm{eq}}\left(r, \Psi\left(X_{3}, \alpha\right), T\right)-\omega d v_{r}\right\}, \\
& \dot{X}_{1}=F_{H}-\frac{3}{T} X_{1}, \\
& \dot{X}_{2}=\frac{3}{T}\left(X_{1}-X_{2}\right), \\
& \dot{X}_{3}=\frac{3}{T}\left(X_{2}-X_{3}\right) .
\end{aligned}
$$

The first equation determines the pressure variations in the proximal tubule in terms of the in and outgoing fluid flows where $F_{f}$ is the single-nephron glomerular filtration rate, reabsorption in the proximal tubule $F_{\text {reab }}$ is assumed to be constant, $F_{H}$ is the flow into the loop of Henle, and $C_{\text {tub }}$ is the elastic compliance of the tubule.

The following two equations describe the dynamics associated with the flow control in the afferent arteriole. Here, $r$ represents the radius of the active part of the vessel and $v_{r}$ is its rate of increase. $d$ is a characteristic time constant describing the damping of the oscillations, $\omega$ is a measure of the mass density of the arteriolar wall, and $P_{\text {av }}$ denotes the average pressure in the active part of the arteriole. $P_{\text {eq }}$ is the value of this pressure for which the arteriole is in equilibrium with its present radius and muscular activation $\Psi$. The expressions for $F_{f}, P_{\text {av }}$, and $P_{\text {eq }}$ involve a number of algebraic equations that must be solved along with the integration of Eqs. (10)-(15).

The remaining equations in the single-nephron model represent the delay $T$ in the TGF regulation. For a more detailed explanation of the model and its parameters see, e.g., Ref. 21.

Thus the mathematical model of vascular-nephron tree we investigate consists of: (i) 12 sets of coupled ODEs describing individual nephrons, (ii) a set of linear algebraic 

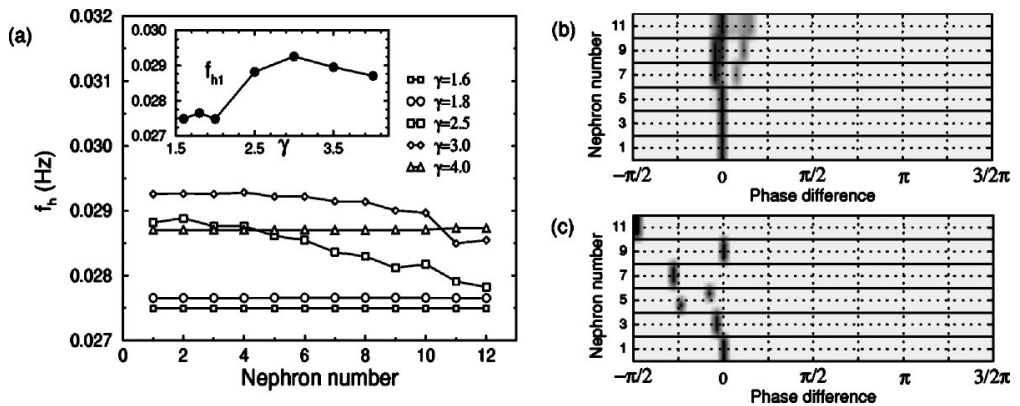

FIG. 9. (a) Slow frequency adjustment with varying vascular coupling $\left(P_{a}=13.3 \mathrm{kPa}, R^{b}=0.002 \mathrm{kPa} \cdot \mathrm{s} / \mathrm{nl}\right)$. Insert shows the variation of $f_{h}$ for the first nephron vs $\gamma$. Phase entrainment of the slow mode at (b) $\gamma$ $=1.6 \mathrm{~mm}^{-1}$ and (c) $\gamma=4.0 \mathrm{~mm}^{-1} ;\left(R^{b}=0.002 \mathrm{kPa} \cdot \mathrm{s} / \mathrm{nl}\right.$ and $P_{a}=13.3 \mathrm{kPa}$ ). Phase differences are calculated with respect to the first nephron.

equations that determines the blood pressure drop from one branching point to another, and (iii) algebraic relations for the vascular interaction.

Depending on the choice of the control parameters, the amplitudes of the pressure oscillations in the nephron tree are found to be different at different positions in the tree. Due to model symmetry, two nephrons connected to the same node have the same oscillation amplitudes. Thus, we can refer to the number of the branching point to describe the amplitude properties. Branching points 2, 3, and 4 may correspond to deep nephrons and branching points 6 and 7 to superficial nephrons. Experimentally, only the pressure oscillations in nephrons near the surface of the kidney have been investigated. However, we suppose that both deep (juxtamedullary) and superficial nephrons can exhibit oscillations in their pressures and flows.

When varying the arterial pressure, different amplitude patterns can be observed in the multinephron models (10)-(15), (8), and (9). For low values of the arterial pressure $P_{a}$, vanishing amplitude of the tubular pressure oscillations can be observed near the top of the tree (i.e., in branching points $5,6,7)$. The nephrons connected to these points operate in a damped regime like the population pools we discussed in the above example. In the individual nephron model, the self-sustained dynamics is bounded by two points of a Hopf bifurcation at $P_{1}=11.48 \mathrm{kPa}$ and $P_{2}=13.86 \mathrm{kPa}$. The calculated value of the mean blood pressure in branching point 5 is lower than $P_{1}$. Hence, neither nephrons connected to this point nor nephrons downstream to it oscillate. As $P_{a}$ increases above $P_{2}$, the upstream nephrons stop to oscillate. For intermediate values of $P_{a}$ there is a cluster of nephrons with self-sustained dynamics in the middle section of the tree. Finally, for high enough values of $P_{a}(>17 \mathrm{kPa})$ only nephrons connected to the branching points 6 and 7 display oscillations because for all other nephrons the blood pressure in the corresponding branching point is too high. Thus, the oscillatory amplitude patterns along a nephron tree have a reasonable explanation in terms of a drop in driving pressure from one branching point to the next, causing a change of the operating regime of the individual nephrons.

\section{Coupling-induced inhomogeneity in vascular-nephron tree}

The nephron tree represents an extended network whose complex dynamics is controlled by a significant number of parameters. To focus our investigations we shall emphasize the generic aspects of cooperative behavior in the network rather than its physiologically relevant properties. From a structural point of view, the object we consider is a population of globally coupled two-mode oscillators. ${ }^{24}$ Besides the relatively slow mode produced by the TGF mechanism with its inherent delay of about $15 \mathrm{~s}$ (associated with the flow of fluid though the loop of Henle), there is a five to six times faster mode arising from the response of the smooth muscles in the arteriolar wall. A typical phenomenon that can be observed in such systems is synchronization. Namely, a certain degree of frequency and/or phase entrainment is expected to occur under variation of appropriate control parameters.

From the classical theory of synchronization it is known that there are two main parameters: The strength (or type) of the interaction and the degree of frequency mismatch. Our first question is, therefore, how the control parameters of the vascular network are related to the synchronization parameters. We have previously shown ${ }^{25}$ that increasing vascular coupling leads to in-phase synchronization while strong hemodynamic interaction can produce anti-phase entrainment. We would expect similar results in our nephron tree. However, the influence of the arterial pressure $P_{a}$ and of the hemodynamic resistances between the neighboring branching points is not trivial since these parameters also affect the natural dynamics of the individual nephrons. Let us perform a few experiments to clarify the situation.

Trial 1 . Weak hemodynamic coupling

With the parameters used in Fig. 8, a choice of the arterial pressure of $P_{a}=13.3 \mathrm{kPa}$ allows all nephrons to be in the oscillatory regime. Here, the hemodynamic resistance has been assumed to be $R^{b}=0.002 \mathrm{kPa} \cdot \mathrm{s} / \mathrm{nl}$. The vascular coupling may then be varied by adjusting of $\gamma$ from $1.6 \mathrm{~mm}^{-1}$ (strong interaction) to $4.0 \mathrm{~mm}^{-1}$ (weak interaction). As defined above, $R^{b}$ denotes the flow resistance between two successive branching points of the vascular tree (see Fig. 8), and the parameter $\gamma$ measures the length constant associated with the exponential decay of the vascular propagated coupling along the arterioles. The frequency distribution among the nephrons is shown in Fig. 9 for the slow $(h)$ oscillatory mode.

For $\gamma=1.6 \mathrm{~mm}^{-1}$, the frequencies of the slow TGF mediated modes are locked at the same value $f_{h}=0.0275 \mathrm{~Hz}$ for all the nephrons. Hence, strong vascular coupling leads to perfect frequency locking along the tree. With decreasing coupling strength, the collective behavior becomes asynchronous. In Fig. 9(a) this is illustrated by the curves for $\gamma=2.5$ and $\gamma=3.0 \mathrm{~mm}^{-1}$. Surprisingly, we find that with further reduction of the coupling $\left(\gamma=4.0 \mathrm{~mm}^{-1}\right)$, all nephrons again 


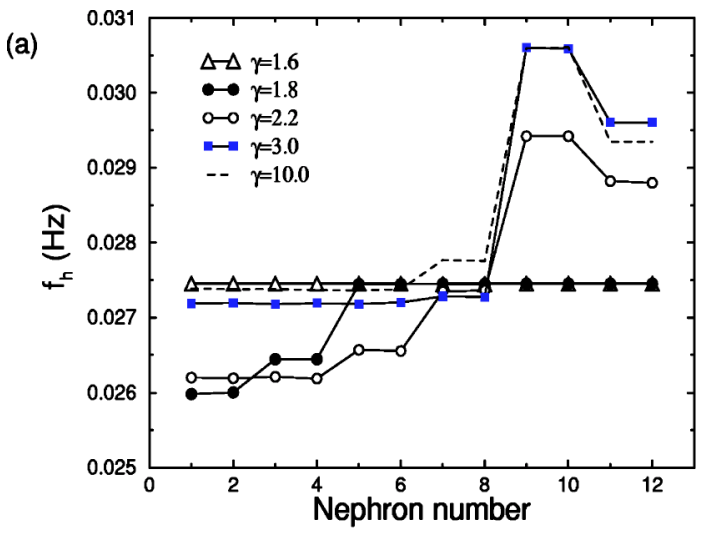

(b)



(c)

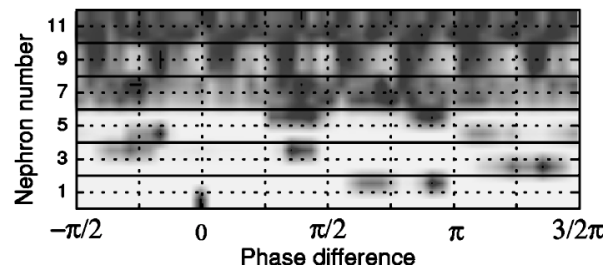

FIG. 10. (a) Slow frequency adjustment for $R^{b}=0.01 \mathrm{kPa} \cdot \mathrm{s} / \mathrm{nl}$ with varying vascular coupling. Phase entrainment of slow dynamics at (b) $\gamma=1.6 \mathrm{~mm}^{-1}$; and (c) $\gamma=3.0 \mathrm{~mm}^{-1} .\left(R^{b}=0.01 \mathrm{kPa} \cdot \mathrm{s} / \mathrm{nl}\right.$ and $\left.P_{a}=13.3 \mathrm{kPa}\right)$.

demonstrate a synchronous state, now at $f_{h} \approx 0.0287 \mathrm{~Hz}$. To explain this, let us consider the phase dynamics [Figs. 9(b) and 9(c)]. In the first synchronous state (strong vascular coupling), in-phase relationships are clearly observed for the slow TGF oscillations [Fig. 9(b)], while the second state (weak vascular interaction) corresponds to out-of-phase synchronization [Fig. 9(c)]. Probably, in this case, the hemodynamic coupling plays the ordering role. The insert in Fig. 9(a) illustrates how the locking frequency within a certain range shifts non-monotonically with varying vascular coupling.

\section{Trial 2. Stronger hemodynamic coupling}

To examine the hypothesis that the hemodynamic interaction mechanism is responsible for the out-of-phase synchronous state at large $\gamma$, we increase the initial hemodynamic coupling to $R^{b}=0.01 \mathrm{kPa} \cdot \mathrm{s} / \mathrm{nl}$ and perform the same experiment with increasing vascular interaction.

Let us focus on the changes in the slow dynamics of the nephrons. For strong vascular coupling $\left(\gamma=1.6 \mathrm{~mm}^{-1}\right)$ all nephrons are frequency locked (Fig. 10). However, this synchronous state is out-of-phase [Fig. 10(b)] in contrast to trial 1 . With decreasing vascular coupling one can observe asynchronous behavior diagnosed both as frequency and phase divergence [Fig. 10(c)]. Note that the pairs of nephrons connected to the same branching point remain synchronous, and the first 6 nephrons operate in synchrony although the distribution looks a little washed out, but remains limited.
However, a globally synchronized state similar to the state shown in Fig. 9 is not achieved even for $\gamma$ $=10.0 \mathrm{~mm}^{-1}$. We conclude that a stronger hemodynamic coupling is unable to synchronize the slow mode oscillations in the whole tree.

With increasing vascular coupling the nephrons are found to synchronize at a lower frequency than in the case of small vascular coupling. This effect cannot be explained solely in terms of the in-phase nature of the vascular coupling. To find an explanation of the observed behavior, let us consider how the vascular coupling influences the natural frequency of the individual nephron. Here, the natural frequency of the $j^{\prime}$ th nephron is understood to be the frequency of a the nephron pressure oscillations in the absence of interaction, but with the same driving pressure as the pressure at a branching point to which the nephron is connected, i.e., $P_{a}=P_{j}^{b}$. Since the vascular coupling acts via the activation potential $\Psi$ of each nephron [Eq. (9)], it can influence the operating regime of the nephron. For in-phase synchronous state, one can estimate this influence via an artificial variation of $\Psi$ in the individual nephron: $\Psi^{\prime}=k \Psi$. Here, $\mu$ is a scaling factor, the stronger the vascular coupling is, the larger $\mu$ will be. Figure 11 illustrates how the nephron frequency depends on $\mu$. It is clearly seen that the oscillation frequency changes in a nonmonotonous way with increasing $\mu$ and becomes lower in average. Thus the vascular coupling
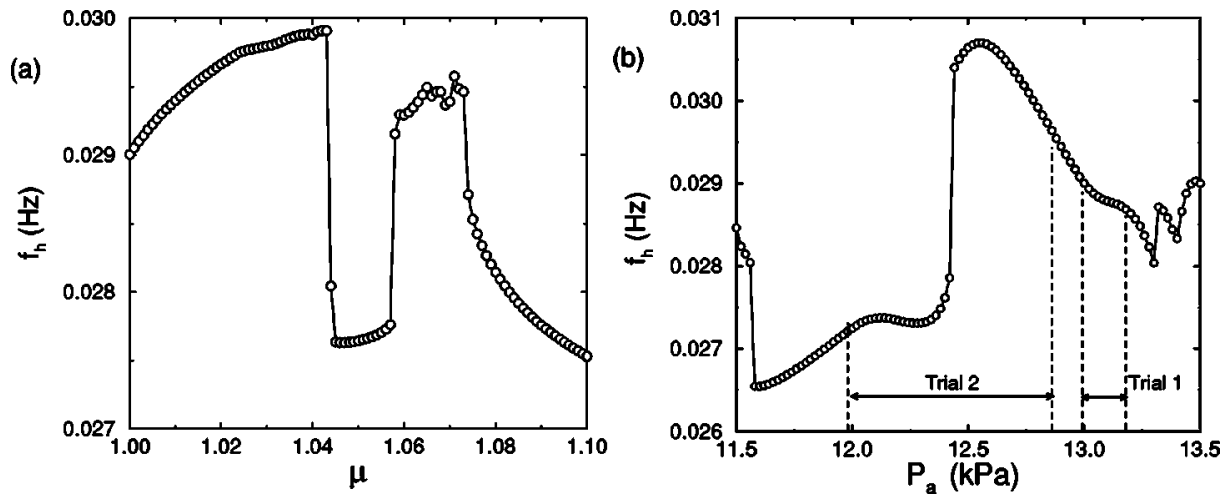

FIG. 11. (a) The strength of the muscular activation affects the oscillation frequency in a nonmonotonic way. $\mu$ is an artificial scaling factor, $\Psi^{\prime}=\mu \Psi$. (b) Frequency of individual nephron as a function of arterial pressure $P_{a}$. 
in general reduces the nephron frequency [at least as long as it is introduced according to (9)].

The influence of the hemodynamic coupling is even more complicated. However, a similar approach can be used. Because the value of the hemodynamic resistance $R^{b}$ is closely related to the drop of arterial pressure along the vascular tree, it can cause essential changes in the oscillatory properties of the individual nephrons.

Figure 11(b) shows how the nephron frequency depends on the driving pressure for the individual nephron. The curve is strongly nonmonotonic and includes pieces of gradual increase, gradual fall, as well as values of abrupt change. Clearly, such behavior is related to the bifurcations of the nephron oscillatory regimes. Thus a variation of the hemodynamic coupling can cause effects that are strongly nonmonotonous and depend on $P_{a}$. In Fig. 11(b), trials 1 and 2 are presented in terms of $P_{j}^{b}$ drop along the vascular tree. It is seen that the first trial corresponds to a relatively small frequency mismatch among the nephrons while stronger coupling $\left(R^{b}=0.01 \mathrm{kPa} \cdot \mathrm{s} / \mathrm{nl}\right)$ leads to a faster drop of arterial pressure along the tree and, hence, to a larger frequency mismatch.

We conclude that:

(i) There is a clear evidence for clustering of oscillators in the vascular tree model. The clustering clearly resembles the similar phenomenon observed in our electronic model (Sec. II) and is obviously governed by the same mechanism;

(ii) due to a coupling induced shift of the operating regime for each nephron, the hemodynamic resistance $R^{b}$ acts in two different ways: As a coupling parameter and as a mismatch parameter; Variation of the parameters of the preglomerular vascular tree produces different conditions under which the nephrons along the tree operate, and this is equivalent to a mismatch between the nephrons;

(iii) similar effects are observed for the vascular propagated coupling. All together, these mechanisms provide for a rather complex response of the system to variations in the coupling strengths.

\section{CONCLUSION}

We considered three examples of coupled oscillator systems where the coupling is mediated though the flow of resources that maintain the oscillatory state in the individual unit.

The electronic example representing a relatively simple chain structure allowed us to demonstrate generic behavioral patterns under competing coupling. Localized (finite sized) clusters of oscillating units that slide up and down the consumption chain in response to changing overall resource supply and coupling-induced inhomogeneity appear to be characteristic phenomena in such systems. For our general model of $N$ identical oscillators, coupling induced inhomogeneity manifests itself either via an asynchronous intra-cluster behavior with a distribution of mean periods resembling the variation of the period of the individual oscillator with the energy supply parameter or (for stronger coupling) via smallsized clusters with out-of-phase synchronization that move along the chain. Note, the resource parameter limited region of self-sustained dynamics appears to be the necessary condition for such type of behavior.

The individual microbiological population pool only displayed a simple Hopf bifurcation. However, different resource delivering environments provide different behavioral patterns. By increasing the lateral resource supply, the chain of population pools could be driven into a state of increasing complexity with clusters of chaotic, frequency synchronized pools. The opposite case of afferent (downstream-only) nutrition provides a finite sized cluster with self-sustained dynamics, outside of which the oscillations die out.

The physiological example of a vascular tree involves a significantly more complicated coupling structure with the flow mediated hemodynamic coupling competing with a vascular propagated coupling of a very different nature. The nonlinearities of the considered physiological system (as specified though the open loop gain curve $\Psi$ for the tubuloglomerular feedback mechanism), only allowed selfsustained oscillations in a finite range of resource supplies. At high and low afferent blood pressures, the individual nephrons displayed stable equilibrium points. Hence, the cluster formation mechanism we observed for a chain of electronic circuits manifests itself in full range. Inside the cluster (or when the cluster occupies the whole vascular tree) we again met the coupling induced inhomogeneity that now activated rather complex patterns due to the complex response of the individual system to external driving.

The type of coupling that we have considered in this paper is likely to be quite common in nature as well as in manmade systems. The generic nature of the resource dependent coupling suggests that it can serve as useful paradigm together with the well-known and widely used approaches assuming interaction via mechanisms unrelated to the resource supply.

\section{ACKNOWLEDGMENTS}

This work was partly supported by INTAS Grant No. 01-2061 and RFBR Grant No. 04-02-16769. D.P. acknowledges support from the Otto Monsteds Fund. O.S. acknowledges support from the Lundbeck Foundation.

${ }^{1}$ A. T. Winfree, The Geometry of Biological Time, 2nd ed. (Springer, New York, 2001).

${ }^{2}$ A. Pikovsky, M. Rosenblum, and J. Kurths, Synchronization: A Universal Concept in Nonlinear Science (Cambridge University Press, Cambridge, 2001).

${ }^{3}$ V. S. Afraimovich and V. I. Nekorkin, "Chaos of traveling waves in a discrete chain of diffusively coupled maps," Int. J. Bifurcation Chaos Appl. Sci. Eng. 4, 631-637 (1994).

${ }^{4}$ Y. Kuramoto, Chemical Oscillations, Waves and Turbulence (Springer, Berlin, 1984).

${ }^{5}$ P. Meda, I. Atwater, A. Goncalves, A. Bangham, L. Orci, and E. Rojas, "The topography of electrical synchrony among $\beta$-cells in the mouse islet of Langerhans,” Q. J. Exp. Psychol. 69, 719-735 (1984).

${ }^{6}$ I. Z. Kiss, Y. Zhai, and J. L. Hudson, "Emerging coherence in a population of chemical oscillators," Science 296, 1676 (2002).

${ }^{7}$ S. Danø, F. Hynne, S. De Monte, F. d'Ovidio, P. G. Sorensen, and H. Westerhoff, "Synchronization of glycolytic oscillations in a yeast cell population," Faraday Discuss. 120, 261-275 (2002). 
${ }^{8}$ D. J. Watts, Small Worlds (Princeton University Press, Princeton, NJ, 1999).

${ }^{9}$ N. Mathias and V. Gopal, "Small worlds: How and why," Phys. Rev. E 63, 021117 (2001).

${ }^{10}$ B. R. Levin, F. M. Stewart, and L. Chao, "Resource-Limited Growth, Competition, and Predation: A Model and Experimental Studies with Bacteria and Bacteriophage," Am. Nat. 111, 3-25 (1977).

${ }^{11}$ J. Stadhouders and G. J. M. Leenders, "Spontaneously Developed MixedStrain Cheese Starters: Their Behaviour towards Phages and their Use in the Dutch Cheese Industry," Neth. Milk Dairy J. 38, 157-181 (1984).

${ }^{12}$ G. Baier, J. S. Thomsen, and E. Mosekilde, "Chaotic Hierarchy in a Model of Competing Populations,” J. Theor. Biol. 165, 593-607 (1993).

${ }^{13}$ D. E. Postnov, A. G. Balanov, and E. Mosekilde, "Synchronization Phenomena in an Array of Population Dynamics Systems," Adv. Complex Syst. 1, 181-202 (1998).

${ }^{14}$ N.-H. Holstein-Rathlou and P. P. Leyssac, "TGF-mediated oscillations in the proximal intratubular pressure: Differences between spontaneously hypertensive rats and Wistar-Kyoto rats," Acta Physiol. Scand. 126, 333339 (1986)

${ }^{15}$ K.-P. Yip, N.-H. Holstein-Rathlou, and D. J. Marsh, "Dynamics of TGFinitiated nephron-nephron interactions in normotensive rats and SHR," Am. J. Physiol. 262, F980-F988 (1992).

${ }^{16}$ N.-H. Holstein-Rathlou, "Synchronization of proximal intratubular pressure oscillation: Evidence for interaction between nephrons," Pfluegers Arch. 408, 438-443 (1987).
${ }^{17}$ A. H. Øien and K. Aukland, "A multinephron model of renal blood flow autoregulation by tubuloglomerular feedback and myogenic response," Acta Physiol. Scand. 143, 71-92 (1991).

${ }^{18}$ G. V. Osipov, A. S. Pikovsky, M. G. Rosenblum, and J. Kurths, "Phase synchronization effects in a lattice of nonidentical Rössler oscillators," Phys. Rev. E 55, 2353 (1997).

${ }^{19}$ R. A. FitzHugh, "Impulses and physiological states in theoretical models of nerve membrane,” Biophys. J. 1, 445-446 (1961).

${ }^{20}$ D. Casellas, F. J. Dupont, T. Kaskel, T. Inagami, and L. C. Moore, "Direct visualization of renin cell distribution in preglomerular vascular tree dissected from rat kidney," Am. J. Physiol. 265, F151-F156 (1993).

${ }^{21}$ M. D. Andersen, N. Carlson, E. Mosekilde, and N.-H. Holstein-Rathlou, Dynamic model of nephron-nephron interaction, in: Membrane Transport and Renal Physiology (Springer, New York, 2001).

${ }^{22}$ E. Mosekilde, Topics in Nonlinear Dynamics: Applications to Physics, Biology and Economic Systems (World Scientific, Singapore, 1996).

${ }^{23}$ M. Barfred, E. Mosekilde, and N.-H. Holstein-Rathlou, "Bifurcation analysis of nephron pressure and flow regulation," Chaos 6, 280-287 (1996).

${ }^{24}$ O. V. Sosnovtseva, A. N. Pavlov, E. Mosekilde, and N.-H. HolsteinRathlou, "Bi-modal oscillations in nephron autoregulation," Phys. Rev. E 66, 061909 (2002).

${ }^{25}$ D. E. Postnov, O. V. Sosnovtseva, E. Mosekilde, and N.-H. HolsteinRathlou, "Cooperative phase dynamics in coupled nephrons," Int. J. Mod. Phys. B 15, 3079-3098 (2001). 